\title{
Pacific
}

Journal of

Mathematics

\section{IDEALS OF FINITE CODIMENSION IN FREE ALGEBRAS AND} THE FC-LOCALIZATION

Amnon Rosenmann And Shmuel Rosset 


\title{
IDEALS OF FINITE CODIMENSION IN FREE ALGEBRAS AND THE FC-LOCALIZATION
}

\author{
Amnon Rosenmann and Shmuel Rosset
}

\begin{abstract}
The topology defined by all finite codimensional right ideals has interesting properties in the case of the free algebra and the group ring of a free group. Its open ideals are precisely the finitely generated essential ones. Finitely generated right ideals are closed and the Leavitt numbers of the associated localization are 1 and $n-1$. The proofs are, for the most part, applications of Schreier's method.
\end{abstract}

This research started when we first became acquainted with the (known) fact that the maximal ring of quotients of a free ring does not have the unique rank property, i.e. there are free modules over it that are of different finite ranks yet they are isomorphic. In fact, since the free ring, and the group ring of a free group have essential right ideals that are free of (countably) infinite rank, it turned out that over the maximal ring of quotients any two finitely generated free modules are isomorphic (see $\S 5$ below). But we noticed also that all the examples we had of finitely generated essential ideals, either in the free ring on $n$ generators over a field $K$ or in $K G$ with $G$ a free group of rank $n$, are of rank $1+m(n-1)$. Our examples were all derived from subgroups of finite index (where Schreier's formula holds!) or by the fractal method, as in our paper [9]. Again, it turns out to be known, and due to Lewin [8], that Schreier's formula extends to ideals of finite codimension in the free ring or the free group ring over a field. From here it is natural to conjecture that if one imitates the construction of the maximal ring of quotients, $Q_{\max }$, but uses right ideals of finite codimension only, then the result would be a ring with the property that two free modules over it are isomorphic if and only if their ranks are congruent modulo $n-1$. This localization we call $Q_{\mathrm{fc}}$. It is defined as $L(L(R))$ where

$$
L(R)=\underset{\lim }{\longrightarrow}\left\{\operatorname{Hom}_{R}(I, R) \mid I \text { is finite-codimensional in } R\right\} \text {. }
$$

Note that a right ideal of finite codimension in the group ring $K G$ is an analogue (and generalization) of the notion of subgroups of finite index in $G$ : to a subgroup $H$ of finite index in $G$ corresponds the right ideal of $K G$ generated by the augmentation ideal of $K H$. 
The critical component in the proof (in $\S 5$ ) that free $Q_{\mathrm{fc}}(R)$ modules, for $R$ a free algebra on $n$ variables or the free group ring of rank $n$ over a field, are isomorphic if and only if their ranks differ by a multiple of $n-1$ is the interesting fact that in such a ring $R$ an essential right ideal is finitely generated if and only if it is of finite codimension in $R$. This is proved in $\S 3$ using strong Schreier bases. It implies that finitely generated essential ideals can only be of rank $1+l(n-1)$.

It turns out that in a finitely generated algebra the linear topology defined by the ideals of finite codimension is a Gabriel topology, i.e. it satisfies the notorious T4 axiom. In fact, if $R$ is a finitely generated algebra over a Noetherian commutative ring $k$ and one defines the fctopology by saying that $I$ is fc if $R / I$ is a finitely generated $k$-module, then this topology satisfies T4 also. We prove this in $\S 1$. In $\S 2$ we give, primarily in order to fix the terminology and notations, a short review of Gabriel's point of view of localization theory (originally started by Johnson [6] and Utumi [11]), torsion theory and injective modules. We also show, in this section, that in the commutative case the fclocalization is interesting for rings of Krull dimension 1.

The fc-topology, being analogous to the profinite topology of group theory, has some similar properties. For example, in $\S 4$ we show that, in analogy to a theorem of $\mathrm{M}$. Hall ([5]), finitely generated ideals in the free algebra or free group rings are closed. For free algebras this is easy enough (and a related result is already in [8]). For free group rings the proof is harder and seems, to us, of interest. It is influenced considerably by an important, and not sufficiently well known, result of Hall (Theorem 5.1 of [4] and Theorem 3.4 of [5]).

It is difficult to decide, in a paper such as this one, in what generality to do things. In $\S 3$, if one defines a Schreier basis and a strong Schreier basis over a commutative ring, the definition is complicated and working with it seems pointless since one loses sight of the main ideas. We therefore elected to work mostly over a field (except in $\S 1)$ and to explain in several places what further generality can be achieved.

1. The Gabriel topology $\mathscr{F}_{\mathrm{fc}}$. If $R$ is a ring then a Gabriel topology on $R$ is a "linear" topology, that is, a topology that has a basis $\mathscr{F}$ of neighborhoods of 0 that consists of right ideals, which satisfy the following special axiom

T4: if $I$ is a right ideal and there exists $J \in \mathscr{F}$ such that for every $x \in J$ the "annihilator" $(I: x):=\{a \in R \mid x a \in I\}$ is in $\mathscr{F}$, then $I \in \mathscr{F}$. 
We refer to Stenström's book [10], Ch. VI for an exhaustive discussion of Gabriel topologies.

Gabriel, in his paper [1], gave a 1-1 correspondence between Gabriel topologies and hereditary torsion theories. Recall that a torsion theory consists of two classes of modules $\mathscr{T}, \mathscr{T}^{\prime}$ such that $M \in \mathscr{T}$ if and only if, for every $M^{\prime} \in \mathscr{T}^{\prime}, \operatorname{Hom}_{R}\left(M, M^{\prime}\right)=0$, and $M^{\prime} \in \mathscr{T}^{\prime}$ if and only if, for every $M \in \mathscr{T}, \operatorname{Hom}_{R}\left(M, M^{\prime}\right)=0$. The class $\mathscr{T}$ of "torsion" modules is closed with respect to quotient modules, direct sums and extensions. If it is also closed with respect to submodules the theory is called hereditary. Conversely, a class of modules closed with respect to all these is the torsion class of a unique torsion theory.

Gabriel's correspondence goes as follows.

Topology $\rightarrow$ Torsion: if $\mathscr{F}$ is a topology then a module $M$ is torsion if for every $x \in M$, its annihilator, $\operatorname{ann}_{R}(x)$, is open, i.e. is in $\mathscr{F}$. It is torsion-free if it does not have any nontrivial torsion submodules.

Torsion $\rightarrow$ Topology: the topology is defined by those ideals $I$ such that $R / I$ is a torsion module.

Now let $R$ be an algebra over a commutative Noetherian ring $k$. Let $\mathscr{F}_{\mathrm{fc}}$ be the linear topology on $R$, whose basic neighborhoods of 0 are the ideals of "finite codimension" in $R$, i.e. those ideals $I$ such that $R / I$ is finitely generated $k$-module.

(1.1) TheOREM. If $R$ is finitely generated as a $k$-algebra, then $\mathscr{F}_{\mathrm{fc}}$ is a Gabriel topology.

Proof. For the sake of completeness we recall T1, T2 and T3. Let $\mathscr{F}$ be a topology. Then

T1: if $I_{1} \subseteq I_{2}$ are right ideals and $I_{1} \in \mathscr{F}$ then $I_{2} \in \mathscr{F}$,

T2: if $I_{1}, I_{2} \in \mathscr{F}$ then $I_{1} \cap I_{2} \in \mathscr{F}$,

T3: if $I \in \mathscr{F}$ and $x \in R$ then $(I: x) \in \mathscr{F}$.

It is clear that $\mathscr{F}_{\mathrm{fc}}$ satisfies $\mathrm{T} 1$ and $\mathrm{T} 2$. To prove T3, let $I \in \mathscr{F}_{\mathrm{fc}}$. If $x \in R$ then the annihilator, in $R$, of its residue class $\bar{x} \in R / I$ is precisely $(I: x)$ and, as $\bar{x} R$ is finitely generated over $k$, we see that $(I: x)$ is indeed in $\mathscr{F}_{\mathrm{fc}}$.

It remains to prove $\mathrm{T} 4$. Instead of proving this directly we apply Gabriel's theorem and show that the torsion theory associated to the fc-topology (see [10], Ch. VI) is in fact a hereditary torsion theory. (This also proves $\mathrm{T} 1, \mathrm{~T} 2$ and $\mathrm{T} 3$ again.) 
It is clear that if $M$ is an $R$-module and an element $u$ has an open annihilator then $u R$ is a $k$-finitely generated $R$-submodule and each of its elements also has an open annihilator. The torsion "radical" $t(\cdot)$ associated to the fc-topology is obviously

\section{$t(M)=$ sum of all $R$-submodules that are finitely generated as $k$-modules.}

A module $M$ is torsion iff $t(M)=M$. As above, it is evident that every submodule of $t(M)$ is in $t(M)$, and that direct sums and quotients of torsion modules are torsion. In order to show that the torsion theory obtained from the fc-topology is indeed a hereditary torsion theory it remains to show that $t(M / t(M))=0$, i.e. the theory is "extension closed" (see [10]). To do this, it is enough to show that if $z \in M$ is such that $z R$ is $k$-finitely generated modulo $t(M)$ then $z R$ is contained in $t(M)$. The assumption on $z R$ means that there exist elements $y_{1}, \ldots, y_{r}$ in $z R+t(M)$ such that, if $U=\sum_{j=1}^{r} y_{j} k$, then $z R \subseteq U+t(M)$. We now use the finite generation of $R$ as a $k$-algebra. Say $R$ is generated by $x_{1}, \ldots, x_{n}$. As $y_{j} \in z R+t(M)$ it follows that

$$
y_{j} x_{l}=\sum_{t} a_{j l t} y_{t}+u_{j l}
$$

where $u_{j l} \in t(M)$ and $a_{j l t} \in k$ for each $j=1, \ldots, r$ and $l=$ $1, \ldots, n$. Hence, if $W$ is a $k$-finitely generated $R$-submodule of $t(M)$ that contains all $u_{j l}$ 's then for every monomial and hence for every element $x$ of $R$,

$$
y_{j} x \in U+W \text {. }
$$

Thus the $R$-submodule generated by the $y$ 's is finitely generated over $k$ (since $k$ is Noetherian) and this implies that the same is true of $z R$. Thus, $z R$ is contained in $t(M)$, as required.

(1.2) EXAMPle. It is instructive to see that in the free ring on infinitely many variables $R=k\left\langle x_{1}, x_{2}, \ldots\right\rangle$ the "augmentation" ideal $I=\sum_{i=1}^{\infty} x_{i} R$ is such that: (i) $I$ is of codimension 1 , (ii) $I^{2}$ is of infinite codimension, (iii) for every $a \in I,\left(I^{2}: a\right)$ contains $I$ and therefore is of codimension $\leq 1$. Of course, $\mathrm{T} 4$ does not hold in the fc-topology of this algebra.

We observe that Theorem (1.1) has the following curious

(1.3) CoROLLARY. If $I, J$ are right ideals of finite codimension in a finitely generated algebra over $k$, then IJ is also of finite codimension. 
Proof. This is true for every Gabriel topology [10], because (IJ : a) $\supseteq J$ for every $a \in I$.

2. Torsion, localization and injective modules. If $\mathscr{F}$ is a Gabriel topology on a ring $R$ and $M$ is a right $R$-module, we let $t_{\mathscr{F}}(M)$ be the sum of all torsion submodules of $M$ relative to the torsion theory associated to $\mathscr{F}$. The following lemma is elementary, but it serves to clarify the picture a little.

(2.1) LEMMA. If $R$ is torsion-free (relative to $\mathscr{F}$ ) then every open ideal is essential.

Proof. Let $I$ be an open ideal. It is enough to show that if $x \neq 0$ then $I \cap x R \neq 0$. But $(I: x)$, being the annihilator of $\bar{x} \in R / I$, is also open, and as $R$ is torsion-free, $(I: x)$ is not contained in $\operatorname{ann}_{R}(x)$, for $\operatorname{ann}_{R}(x)$ is not open. Thus $x(I: x) \neq 0$ and $x(I: x) \subseteq$ $I \cap x R$.

The lemma explains the fact that essential ideals commonly occur in this domain. Curiously enough, the topology defined by all essential right ideals may not satisfy $\mathrm{T} 4$. Its torsion theory is evidently the "singular" torsion

$$
Z(M)=\left\{x \in M \mid \operatorname{ann}_{R}(x) \text { is essential }\right\} .
$$

If the ring is non-singular, i.e. $Z(R)=0$, then this topology does satisfy T4. There is a topology, the "dense" topology, which is the largest topology relative to which $R$ is torsion-free. It is the set of (essential) right ideals $I$, such that for every $x \in R$, the left annihilator of $(I: x)$ is trivial.

The localization associated to a Gabriel topology $\mathscr{F}$ is defined as follows. If $M$ is a right $R$-module, let

$$
M_{(\mathscr{F})}=\underset{I \in \mathscr{F}}{\lim } \operatorname{Hom}_{R}(I, M) .
$$

It is an $R_{(\mathscr{F})}$-module and an $R$-module in an obvious way and there is a canonical map $M \rightarrow M_{(\mathscr{F})}$ whose kernel (see [10], p. 196) is $t_{\mathscr{F}}(M)$. Moreover, if $M$ is torsion then $M_{(\mathscr{F})}=0$. Now define

$$
M_{\mathscr{F}}=\underset{I \in \mathscr{F}}{\lim } \operatorname{Hom}_{R}\left(I, M_{(\mathscr{F})}\right) \text {. }
$$

One proves that $M_{\mathscr{F}}=(M / t(M))_{(\mathscr{F})}$. In particular, if $M$ is torsionfree then $M_{\mathscr{F}}=M_{(\mathscr{F})}$, i.e. the second step of passing from $M_{(\mathscr{F})}$ to $M_{\mathscr{F}}$ is redundant. 
(2.2) ExAmples. (i) The "maximal" localization, associated to the dense topology, is a very important object. It is denoted by $M_{\max }$ and the ring by $Q_{\max }(R)$ or $R_{\max }$. If $\mathscr{F}$ is any Gabriel topology on $R$ such that $R$ is torsion-free relative to $\mathscr{F}$ then all open ideals in $\mathscr{F}$ are dense, i.e. $\mathscr{F}$ is contained in the dense topology, and the natural map $R_{\mathscr{F}} \rightarrow R_{\max }$ is an embedding. If $R$ is non-singular this localization was described by Johnson [6], the general case is due to Utumi [11].

(ii) If $R$ is finitely generated over a Noetherian commutative ring $k$ then the fc-topology is Gabriel as shown in (1.1). Thus, if $R$ does not have right ideals that are finitely generated as $k$-modules then the localization $R_{\mathrm{fc}}$ is contained, canonically, in $R_{\max }$.

It is useful to have criteria that ensure that $M_{(\mathscr{F})}=M_{\mathscr{F}}$. One useful criterion has already been mentioned, and it is that $M$ be torsion-free. Another one is:

(2.3) LEMмA. If all the ideals in $\mathscr{F}$ are projective as $R$-modules then, for every module $M, M_{\mathscr{F}}=M_{(\mathscr{F})}$.

Proof. The exact sequence

$$
0 \rightarrow t(M) \rightarrow M \rightarrow M / t(M) \rightarrow 0
$$

induces

$$
\begin{aligned}
0 & \rightarrow \operatorname{Hom}_{R}(I, t(M)) \rightarrow \operatorname{Hom}_{R}(I, M) \\
& \rightarrow \operatorname{Hom}_{R}(I, M / t(M)) \rightarrow \operatorname{Ext}_{R}^{1}(I, t(M))=0
\end{aligned}
$$

where $\operatorname{Ext}_{R}^{1}(I,-)=0$ since $I$ is projective. As the direct limit is exact and $t(M)_{(\mathscr{F})}=0$ (see [10]) we get an isomorphism

$$
M_{(\mathscr{F})}=\underset{\lim }{\longrightarrow} \operatorname{Hom}_{R}(I, M) \rightarrow \underline{\lim } \operatorname{Hom}_{R}(I, M / t(M))=M_{\mathscr{F}} .
$$

This lemma is useful when $R$ is a free algebra or a free group ring over a field, since then ideals are free.

If $I$ is an open ideal in the topology $\mathscr{F}$ then the exact sequence

$$
0 \rightarrow I \rightarrow R \rightarrow R / I \rightarrow 0
$$

gives rise, when $\operatorname{Hom}_{R}(-, R)$ acts on it, to

$$
\begin{aligned}
0 & \rightarrow \operatorname{Hom}_{R}(R / I, R) \rightarrow \operatorname{Hom}_{R}(R, R) \\
& \rightarrow \operatorname{Hom}_{R}(I, R) \rightarrow \operatorname{Ext}_{R}^{1}(R / I, R) \rightarrow 0 .
\end{aligned}
$$

Identifying $\operatorname{Hom}_{R}(R, R)$ with $R$ and passing to the limit, over $I$, gives

$$
0 \rightarrow t_{\mathscr{F}}(R) \rightarrow R \rightarrow R_{(\mathscr{F})} \rightarrow \underline{\lim _{\longrightarrow}} \operatorname{Ext}_{R}^{1}(R / I, R) \rightarrow 0
$$


Here we identified $t(R)$ with

$$
\underset{\lim }{\longrightarrow} \operatorname{Hom}_{R}(R / I, R) \text {. }
$$

Assume now that $R$ is torsion-free. Then we know that $R_{(\mathscr{F})}=R_{\mathscr{F}}=$ $Q_{\mathscr{F}}(R)$ and we get the exact sequence

$$
0 \rightarrow R \rightarrow Q_{\mathscr{F}}(R) \rightarrow \underset{\lim }{\longrightarrow} \operatorname{Ext}_{R}^{1}(R / I, R) \rightarrow 0 .
$$

We can use this exact sequence to analyze $Q_{\mathrm{fc}}(R)$, when $R$ is a commutative, finitely generated, Cohen-Macaulay algebra over a field.

(2.5) Proposition. With these assumptions, if $\operatorname{dim}(R) \geq 2$ then $Q_{\mathrm{fc}}(R)=R$. If $\operatorname{dim}(R)=1$ then

$$
\stackrel{\lim }{\longrightarrow} \operatorname{Ext}_{R}^{1}(R / I, R)
$$

is the direct sum of local cohomology groups $H_{\mathfrak{m}}^{1}(R)$, where $\mathfrak{m}$ runs on the maximal spectrum of $R$.

Proof. If $I$ is a finite codimensional ideal in $R$ then clearly the ideals associated with $R / I$ are maximal ideals. For every maximal ideal $\mathfrak{m}$

$$
R_{\mathfrak{m}} \otimes_{R} \operatorname{Ext}_{R}^{1}(R / I, R) \approx \operatorname{Ext}_{R_{\mathfrak{m}}}^{1}\left(R_{\mathfrak{m}} / I R_{\mathfrak{m}}, R_{\mathfrak{m}}\right),
$$

and $I R_{\mathfrak{m}}$ is either $R_{\mathfrak{m}}$ or $\mathfrak{m} R_{\mathfrak{m}}$-coprimary if $\mathfrak{m} \in \operatorname{ass}(I)$. Since $R_{\mathfrak{m}}$ is Cohen-Macaulay, it follows that if $\operatorname{dim}(R) \geq 2$ then $R_{\mathfrak{m}} \otimes_{R}$ $\operatorname{Ext}_{R}^{1}(R / I, R)=0$ for every maximal ideal and so $\operatorname{Ext}_{R}^{1}(R / I, R)=0$. Since this is true for every $I$ we see that $Q_{\mathrm{fc}}(R)=R$ in this case. If $\operatorname{dim}(R)=1$ then

$$
\stackrel{\lim }{\longrightarrow} \operatorname{Ext}_{R}^{1}(R / I, R)
$$

is clearly the direct sum, on all of the maximal spectrum of $R$, of the groups

$$
\underset{n}{\lim } \operatorname{Ext}_{R_{\mathfrak{m}}}^{1}\left(R_{\mathfrak{m}} / \mathfrak{m}^{n} R_{\mathfrak{m}}, R_{\mathfrak{m}}\right)
$$

and this is known (see e.g. [2]) to be the local cohomology group $H_{\mathfrak{m}}^{1}(R)$.

The local cohomology groups are of great interest. They are tools in duality theory and, for example, when $R$ is a local Gorenstein ring of dimension 1 with residue field $k$ then $H_{\mathfrak{m}}^{1}(R)$ is an injective hull of $k$.

The appearance of injective modules is not accidental. Returning now to the general case of a ring $R$ and a Gabriel topology $\mathscr{F}$ 
on it, one says that a module $E$ is $\mathscr{F}$-injective if for every injection $0 \rightarrow M^{\prime} \rightarrow M$ whose cokernel is torsion the restriction map $\operatorname{Hom}_{R}(M, E) \rightarrow \operatorname{Hom}_{R}\left(M^{\prime}, E\right)$ is onto. It is then known (see [10], Ch. IX) that if $M$ is a torsion-free module then the canonical map $M \rightarrow M_{\mathscr{F}}$ is an $\mathscr{F}$-injective envelope. Since injective modules are of such wide applicability this connection further justifies the study of various localizations.

3. Free algebras and free group rings. If $R$ is an integral domain (such as the free non-commutative algebra over a Noetherian commutative integral domain $k$ ), a right ideal $I$ of finite codimension is essential because every right ideal is not finitely generated as a $k$ module and hence must intersect $I$ non-trivially (this also follows from Lemma (2.1)). From the Schreier method it is known (see e.g. [8], where everything is done over a field), that in a finitely generated algebra over $k$, an ideal of finite codimension is finitely generated. We prove now that, in finitely generated "free algebras", being finite codimensional is the same as being finitely generated and essential. As mentioned before, we restrict the discussion to the case of a base field; the same result over a commutative Noetherian domain is much more cumbersome but not more difficult in principle.

We start by defining a Schreier basis and a strong Schreier basis, assuming that $K$ is a field. So let $R$ be a finitely generated $K$-algebra, with a given set of generators $x_{1}, \ldots, x_{n}$ and $I$ a right ideal of $R$.

(3.1) Definition. A Schreier basis for $R / I$, relative to a given set of generators $x_{1}, \ldots, x_{n}$, is defined as a set $B$ of $K$-linearly independent monomials in $x_{1}, \ldots, x_{n}$, that spans a subspace $V$ in $R$ that is complementary to $I$ (that is, $I+V=R, I \cap V=0$ ), and which is closed to "taking initials". This means that every element $b$ in $B$ has a presentation $b=x_{i_{1}} \cdots x_{i_{r}}\left(i_{j} \in\{1, \ldots, n\}\right.$ for $\left.j=1, \ldots, r\right)$ such that all its initial segments $x_{i_{1}} \cdots x_{i_{s}}, 0 \leq s \leq r$, are also in $B$ and are distinct. Here the empty word is taken as the unit element 1.

A strong Schreier basis is a Schreier basis that has the additional property that every monomial $x_{i_{1}} \cdots x_{i_{m}}$ of "length" $m$ lies in $\mathrm{I}+$ (the linear span of the elements of length $\leq m$ in $B$ ). Here the length of an element of $B$ is the minimal length of a presentation of it which is closed to taking initials.

REMARK. It seems that the definition of a Schreier basis that is usually given does not include the requirement we make for "strong Schreier basis". On the other hand, the Schreier bases that are ob- 
tained by well ordering techniques are more than "strong" (see e.g. [8]). Our Lemma (3.2) shows that one can construct strong Schreier bases in a very simple inductive way.

The way the Schreier basis gives a generating set for $I$ is well known. In the notation above, if $\pi: R \rightarrow V$ denotes the projection onto $V$ (with kernel $I$ ) then $I$ is generated by the set

$$
\left\{b x_{i}-\pi\left(b x_{i}\right) \mid b \in B, i=1, \ldots, n\right\} .
$$

(3.2) LEMMA. If $R$ is a finitely generated algebra over a field $K$ and $I$ is a right ideal then for any finite generating set $\left\{x_{1}, \ldots, x_{n}\right\}$ for $R$ there is a strong Schreier basis for $R / I$ relative to this set.

Proof. We construct inductively such a basis $B$. If $I=R$ let $B=\varnothing$. If not, let $B_{0}=\{1\}$. For clarity we define $B_{1}$ explicitly. If $I+K \cdot 1=R$ then $B_{1}=B_{0}$. If not, then $I+K \cdot 1$ does not contain the subspace $\sum_{i=1}^{n} K \cdot x_{i}$. Let $B_{1}^{\prime}$ be a maximal subset of $\left\{x_{1}, \ldots, x_{n}\right\}$ which is linearly independent modulo $I+K \cdot 1$. Then $B_{1}=B_{0} \cup B_{1}^{\prime}$. Assuming now that $B_{n}^{\prime}$ and $B_{n}$ have been defined, let $B_{n+1}^{\prime}$ be a maximal subset of the set of monomials (of length $n+1$ )

$$
\left\{b x_{i} \mid b \in B_{n}^{\prime}, i=1, \ldots, n\right\},
$$

which is linearly independent modulo $I+\sum_{b \in B_{n}} K \cdot b$. Then $B_{n+1}=$ $B_{n} \cup B_{n+1}^{\prime}$. If $B_{n+1}^{\prime}=\varnothing$, the process stops at this stage. Finally, define $B=B_{n}$ if $B_{n+1}^{\prime}=\varnothing$, and if the process does not stop after finitely many steps let

$$
B=\bigcup_{j=0}^{\infty} B_{j} .
$$

It is clear that, as constructed, $B$ is a linearly independent set that spans a subspace $V$ such that $I \cap V=0$. It is also clear that $B$ is closed with respect to taking initial segments. We now prove, by induction, the "strong Schreier" property of $B$-that every monomial of length $n$ is in the linear span of $B_{n}$ modulo $I$. For $n=0$ this is trivial. Assuming the result for monomials of length $\leq n-1 \quad(n \geq 1)$, let $g$ be a monomial of length $n$. Then $g=g^{\prime} x_{i}$ for some $g^{\prime}$ of length $n-1$ and some $i$. We denote the linear span of $B_{j}$ by $V_{j}$. Then we know, from the inductive hypothesis, that $g^{\prime} \in I+V_{n-1}$. From the maximality of $B_{n}^{\prime}$, it is seen that for every $i=1, \ldots, n$, if $h$ is a monomial of $V_{n-1}$ then $h x_{i}$ is in $I+V_{n}$. In particular, $I+V=R$ and the proof is complete. 
(3.3) THEOREM. If $R$ is a free algebra $K\left\langle x_{1}, \ldots, x_{n}\right\rangle$ or the group ring $K G$ of a finitely generated free group then a right ideal is finite codimensional if, and only if, it is finitely generated and essential.

Proof. By the above, it remains to prove that a finitely generated and essential ideal is finite codimensional.

We use the letters $x_{1}, \ldots, x_{n}$ to denote both the free variables in the free algebra as well as a basis of the free group.

If $n=1$ then $K\langle x\rangle$ and $K G$ are principal ideal domains and the result is trivial. Thus, we assume that $n \geq 2$.

Let $I$ be a finitely generated essential ideal in the ring, and let $B$ be a strong Schreier basis for $R / I$, relative to the generating set $x_{1}, \ldots, x_{n}$ if $R=K\left\langle x_{1}, \ldots, x_{n}\right\rangle$, or the set $x_{1}, x_{1}^{-1}, \ldots, x_{n}, x_{n}^{-1}$ if $R=K G$. We must prove that $B$ is finite. Assume not. Let $V$ be the linear span of $B$, so that $I+V=R$ is a direct sum, and let $\pi: R \rightarrow V$ be the corresponding projection onto $V$, "parallel" to $I$. According to Lewin [8], $I$ is freely generated by the set

$$
\left\{b x_{i}-\pi\left(b x_{i}\right) \mid i=1, \ldots, n, b \in B, b x_{i}-\pi\left(b x_{i}\right) \neq 0\right\} .
$$

Since $I$ is assumed to be finitely generated, this last set must be finite. It follows, in particular, that there exists $r \geq 1$, such that for every $b \in B$ of reduced length $\geq r$, all the monomials $b x_{i}, i=1, \ldots, n$, are also in $B$. If $R=K\left\langle x_{1}, \ldots, x_{n}\right\rangle$ this means that all monomials of length $\geq r$, that have an initial segment of length $r$ which is in $B$, are also in $B$. In other words, if $b \in B$ is a monomial of length $r$ then the right ideal $b R$ intersects $I$ trivially (as follows from the properties of Schreier basis). This contradicts the essentiality of $I$ and completes the proof in the case of a free algebra.

If $R=K G$ then an argument similar to the above works but it may be instructive to give the following direct proof. We claim that for every $b \in B$ of reduced length $\geq r+1$, every $b x_{i}^{-1}, i=1, \ldots, n$, is also in $B$. If $b=b^{\prime} x_{i}$, with $l\left(b^{\prime}\right)=l(b)-1, l$ denoting the reduced length, then $b x_{i}^{-1}=b^{\prime}$ is in $B$ as it is an initial segment of $b$. If $b x_{i}^{-1}$ satisfies $l\left(b x_{i}^{-1}\right)=l(b)+1$ (i.e. $b$ does not end in $x_{i}$ ) write

$$
b x_{i}^{-1}=\sum_{j} a_{j} b_{j}(\bmod I), \quad a_{j} \in K, b_{j} \in B .
$$

As $B$ is a strong Schreier basis, we know that $l\left(b_{j}\right) \leq l\left(b x_{i}^{-1}\right)$ for every $j$. We now analyze the equality

$$
b=\sum_{j} a_{j} b_{j} x_{i}(\bmod I)
$$


in light of everything we know. Since the Schreier basis is strong, when $l\left(b_{j}\right)<l(b)-1$ and $b_{j} x_{i}$ is written out as a linear combination of elements of $B(\bmod I)$, these elements are of length $<l(b)$. Thus, in the sum $(*)$ they must all cancel out. If $l\left(b_{j}\right) \geq l(b)-1$ then $l\left(b_{j}\right) \geq r$ and we know that $b_{j} x_{i}$ is in $B$. Note that by all the above, as $b \in B$, it follows from the properties of a Schreier basis that the equality $(*)$ implies that $b$ is equal to one of the summands $b_{j} x_{i}$ on the right. But if $b=b_{j} x_{i}$ then, of course, $b x_{i}^{-1}=b_{j} \in B$. This proves that every group element, which when written in reduced form has an initial segment $b \in B$ of length $>r$, is in $B$.

We can now complete the proof in a way that is analogous to the case of $K\langle x\rangle$ above. Let $b_{1}$ and $b_{2}$ be elements of $B$ of the same length $s$, $s>r$, that end differently, i.e. when written in reduced form their last letters are different. We claim that the principal right ideal generated by $b_{1}+b_{2}$ has trivial intersection with $I$. Indeed, if $0 \neq \alpha \in K G$ let $g \in G$ be a monomial of maximal length appearing in $\alpha$. Then it is clear that either $b_{1} g$ or $b_{2} g$ is of length $s+l(g)$ and thus, as we showed above, is in $B$. This last element is linearly independent $(\bmod I)$ of all other group elements occurring in $\left(b_{1}+b_{2}\right) \alpha$. So, we see that

$$
I \cap\left(b_{1}+b_{2}\right) R=\{0\},
$$

which contradicts the essentiality of $I$.

It follows that $B$ is finite, i.e. $I$ is of finite codimension, as claimed.

It is well known that in a finitely generated free group a finitely generated normal subgroup is of finite index. This result is analogous to (and is also implied by) the following consequence of the above theorem.

(3.4) Corollary. Let $R$ be as in (3.3). Then a two-sided ideal of $R$ is finitely generated (as a right ideal) if, and only if, it is finite codimensional.

Proof. This follows from (3.3) since a nontrivial 2-sided ideal in an integral domain is essential.

4. Finitely generated ideals. The fc-topology of the group ring is evidently an analogue of the profinite topology of the group. In 1950 M. Hall proved ([5]) that a finitely generated subgroup of a free group is closed in the profinite topology. He did this by proving that if $x \in G-H$, where $G$ is free and $H$ is a finitely generated subgroup, 
then there is a subgroup of finite index containing $H$ which does not contain $x$. His proof was an application of a result of Hall and Radò ([3]) which is, in a sense, a converse to Schreier's theorem. They proved that a prefix-closed set $B$ (satisfying another restriction, which disappears when $B$ is finite) in a free group is a Schreier system for an appropriate subgroup. In this section we show that a similar result holds for the free algebra and the free group ring.

We start by showing that the following analogue of Theorem 3.3 of Hall's paper [5] is true.

(4.1) Proposition. Let $\mathscr{F}$ be a linear topology on the ring $R$. Then a right ideal is closed in this topology if and only if it is the intersection of all open ideals that contain it.

Proof. Since an open ideal is closed (as its complement is a union of its cosets, which are open), the condition is clearly sufficient.

Conversely, if $I$ is a closed ideal and $z \notin I$, then there exists an open ideal $J$ such that $(J+z) \cap I=\varnothing$. Let $L$ be the ideal $J+I$. As $L$ contains $J$, it is open. From $(J+z) \cap I=\varnothing$, it follows that $z \notin L$. Thus, $I$ is equal to the intersection of the open ideals that contain it.

Now let $G$ be the free group on $x_{1}, \ldots, x_{n}$. Let $B$ be a Schreier system, i.e. a prefix-closed set of elements of $G$ (written in reduced form relative to the generators $\left.x_{1}, \ldots, x_{n}\right)$. For each $i, 1 \leq i \leq n$, define

$$
B^{(i+)}=\left\{b \in B \mid b x_{i} \in B\right\},
$$

and

$$
B^{(i-)}=\left\{b \in B \mid b x_{i}^{-1} \in B\right\} .
$$

Recall that if $I$ is a right ideal of $R=K G$ and $B$ is a Schreier basis for $R / I$ then we denoted by $\pi(\alpha)$ the coset representative of $\alpha \in R$ (relative to $B$ ). The vector space spanned by $B$ is denoted by $\operatorname{sp}(B)$.

(4.2) THEOREM. If $I$ is a right ideal of $K G$ and $B$ is a Schreier basis for $K G / I$ then for every $i, 1 \leq i \leq n$, the set

$$
\left\{\pi\left(b x_{i}\right) \mid b \in B\right\}
$$

is a $K$-basis for $\operatorname{sp}(B)$.

Conversely, assume $B$ is a Schreier system and

$$
\pi:\left\{b x_{i} \mid b \in B, i=1, \ldots, n\right\} \rightarrow \operatorname{sp}(B)
$$


is a function which satisfies

(i) $\pi(b)=b$ if $b=b^{\prime} x_{i}$ with $b, b^{\prime} \in B$, and

(ii) for every fixed $i, 1 \leq i \leq n$, the set $\left\{\pi\left(b x_{i}\right) \mid x \in B\right\}$ is $a$ $K$-basis for $\operatorname{sp}(B)$.

Then

$$
\left\{b x_{i}-\pi\left(b x_{i}\right) \mid b \in B-B^{(i+)}, i=1, \ldots, n\right\}
$$

freely generates a right ideal $I$, and $B$ is a Schreier basis for $K G / I$.

Proof. Let $I$ be a right ideal of $K G$ and let $B$ be a Schreier basis for $K G / I$. Suppose that $\sum_{j} a_{j} \pi\left(b_{j} x_{i}\right)=0, a_{j} \in K, b_{j} \in B$. Then $\sum_{j} a_{j} b_{j} x_{i} \in I$, and hence also $\sum_{j} a_{j} b_{j} \in I$. But since the $b_{j}$ are linearly independent $(\bmod I)$ then $a_{j}=0$ for each $j$ and we see that the set $\left\{\pi\left(b x_{i}\right) \mid b \in B\right\}$ is linearly independent for every $i, 1 \leq$ $i \leq n$. Moreover, this set also generates $\operatorname{sp}(B)$. For if $b \in B$, then $\pi\left(b x_{i}^{-1}\right)=\sum_{j} a_{j} b_{j}, a_{j} \in K, b_{j} \in B$, and hence $b=\pi\left(\sum_{j} a_{j} b_{j} x_{i}\right)=$ $\sum_{j} a_{j} \pi\left(b_{j} x_{i}\right)$.

For the converse, we will show first that, with the given assumptions, the elements

$$
u_{b, i}:=b x_{i}-\pi\left(b x_{i}\right),
$$

with $b$ running on $B-B^{(i+)}$ and $i=1, \ldots, n$, are independent over the ring, i.e. they generate a free module of which they are a basis. To do this, we need to show that if $\alpha_{b, i} \in K G$, where only finitely many of them are $\neq 0$, and $\sum u_{b, i} \alpha_{b, i}=0$ then $\alpha_{b, i}=0$ for every $b \in B-B^{(i+)}$ and every $i=1, \ldots, n$. Each $\alpha_{b, i}$ is a sum, with coefficients in $K$, of group elements and, as in $\S 3$, we use the length of the group elements. The simplest case is when a group element $g$ of maximal length (among all those that appear in all $\alpha_{b, i}$ above) appears in an $\alpha_{b_{0}, i_{0}}$ and is such that its first letter is not $x_{i_{0}}^{-1}$. Then we claim that the element $b_{0} x_{i_{0}} g$, which comes from $u_{b_{0}, i_{0}} \alpha_{b_{0}, i_{0}}$, cannot cancel. This is because $b_{0} x_{i_{0}}$ is not a prefix of any of the elements $b x_{i}, b \in B-B^{(i+)}, i=1, \ldots, n$, and $(b, i) \neq\left(b_{0}, i_{0}\right)$, and of any of the group elements in $\pi\left(b x_{i}\right)$ (without any exception, even including $\left.\pi\left(b_{0} x_{i_{0}}\right)\right)$.

It remains to prove that the $\alpha_{b, i}$ vanish even when, for every $i=$ $1, \ldots, n$ and $b \in B-B^{(i+)}$, every group element of maximal length that appears in $\alpha_{b, i}$ starts with $x_{i}^{-1}$. (Here, as before, an element of "maximal length" is one which is longest among all group elements that appear in all $\left.\alpha_{b, i}.\right)$ 
Fix $i$ such that a group element of maximal length appears in $\alpha_{b, i}$. For each $b \in B-B^{(i+)}$ write

$$
\pi\left(b x_{i}\right)=\gamma_{b, i}+\delta_{b, i}
$$

where $\gamma_{b, i}$ is in the linear span of $B^{(i-)}$ and $\delta_{b, i}$ in that of $B-B^{(i-)}$. We claim that the set

$$
\left\{\delta_{b, i} \mid b \in B-B^{(i+)}\right\}
$$

is linearly independent. For suppose that

$$
\sum_{b \in B-B^{(i+)}} \lambda_{b} \delta_{b, i}=0,
$$

with $\lambda_{b} \in K$. This implies that

$$
\sum_{b \in B-B^{(i+)}} \lambda_{b} \pi\left(b x_{i}\right)=\sum_{b \in B-B^{(i+)}} \lambda_{b} \gamma_{b, i} .
$$

However, each element of $B^{(i-)}$ is of the form $b x_{i}$ where $b \in B^{(i+)}$. Hence the span of $B^{(i-)}$ is equal to that of the elements of $\pi\left(b x_{i}\right)=b x_{i}, b \in B^{(i+)}$, and $\sum_{b \in B-B^{(i+)}} \lambda_{b} \gamma_{b, i}$ can be written as $\sum_{b \in B^{(i)}} \mu_{b} \pi\left(b x_{i}\right)$. Substituting the last expression in the right-hand side of $(*)$ we get a linear dependence among the elements $\pi\left(b x_{i}\right)$, $b \in B$. Since we assumed that they are linearly independent, the $\lambda_{b}, b \in B-B^{(i+)}$, must all vanish.

It remains to show that in the sum $\sum u_{b, j} \alpha_{b, j}$, where $b \in B-B^{(i+)}$ and $j=1, \ldots, n$, there appears a summand, this time of the form $\pi\left(b x_{i}\right)$ times a group element of maximal length, that does not cancel. Here $i$ is the index chosen above. Let $g$ be a group element of maximal length that appears in $\alpha_{b, i}, b \in B-B^{(i+)}$. Then we know that $g$ starts with $x_{i}^{-1}$ (i.e. $g=x_{i}^{-1} g^{\prime}$ when written in reduced form). Our purpose is to show that for some group element $h$ appearing in some $\delta_{b, i}\left(b \in B-B^{(i+1)}\right)$ the product $h g$ does not cancel.

To do this, we will use the notion of "distance" from the Schreier set $B$. If $z \in G$ is of reduced length $n$ and a maximal prefix of it in $B$ is of length $m$ then the distance of $z$ from $B$ is defined to be $n-m$. We denote this function by $d(B, z)$. By the linear independence of the $\delta_{b, i}$, it is clear that there is a group element $h$ that appears in some $\delta_{b, i}\left(b \in B-B^{(i+)}, i\right.$ fixed as above) such that in the sum $\sum_{\left.b \in B-B^{(i)}\right)} \delta_{b, i} \alpha_{b, i}$, the element $h g$ does not cancel. The distance of $h g$ from $B$ is evidently the length of $g$. We claim that 
this element does not cancel also in the sum $\sum_{b \in B-B^{(i+)}} u_{b, i} \alpha_{b, i}$. For write each $u_{b, i}$ as

$$
u_{b, i}=b x_{i}-\gamma_{b, i}-\delta_{b, i}
$$

In the product $b x_{i} g$, the $x_{i}$ cancels, so the distance from $B$ of $b x_{i} g$ $\left(b \in B-B^{(i+)}\right)$ is less than $d(B, h g)$. This shows that $h g$ does not appear in any $b x_{i} g$. But as $g$ is of maximal length in $\alpha_{b, i}, h g$ cannot appear also in any $b x_{i} \alpha_{b, i}$. Similarly, as for every element appearing in $\gamma_{b, i}, b \in B-B^{(i+)}$, its right multiple by $x_{i}^{-1}$ is in $B$, the distance from $B$ of this element multiplield by $g$ is less than $d(B, h g)$. This completes the proof that $h g$ does not cancel in $\sum_{b \in B-B^{(i+)}} u_{b, i} \alpha_{b, i}$. The proof will be finished once we show that the element $h g$ does not appear in any $u_{b, j} \alpha_{b, j}$ for $j \neq i$ and $b \in B-B^{(j+)}$. This follows directly from our assumption that every group element of maximal length appearing in $\alpha_{b, j}$ starts with $x_{j}^{-1}$. This completes the proof that the $u_{b, j}, b \in B-B^{(j+)}, j=1, \ldots, n$, are independent over $R=K G$.

It only remains to verify that $B$ is a Schreier basis for $R / I$ when $I$ is the right ideal generated by the elements $u_{b, j}$ as above. To show that $I+\operatorname{sp}(B)=R$, we use induction on the distance from $B$. Recall that for every group element $g$ we defined its distance from $B, d(B, g)$. If $r \in R$ is a combination $r=\sum_{i} \lambda_{i} g_{i}, \lambda_{i} \in K$, we define $d(B, r)$ as $\max _{i} d\left(B, g_{i}\right)$. If $r \in R$ is such that $d(B, r)=0$ then, of course, $r \in \operatorname{sp}(B)$. Assuming that elements of distance $\leq n$ from $B$ are in $I+\operatorname{sp}(B)$, let $r$ be of distance $n+1$ from $B . r$ is a sum of group elements (with coefficients), each of which is of distance $\leq n+1$ from $B$. So, it suffices to show that every group element of the form $b g$, with $g$ of length $n+1$ and $b \in B$, is in $I+\operatorname{sp}(B)$. If $g=x_{i} g^{\prime}$ then $b g=b x_{i} g^{\prime}$ and $b x_{i} \in I+\operatorname{sp}(B)$, for either $b x_{i} \in B$ or $b x_{i}=u_{b, i}+\pi\left(b x_{i}\right)$. Thus, $b g$ is contained in $I+$ the subspace spanned by the group elements of distance $\leq n$ from $B$, and these last are covered by the induction hypothesis. If $g=x_{i}^{-1} g^{\prime}$, and $b x_{i}^{-1} \notin B$, then since we assumed that the set $\pi\left(b x_{i}\right), b \in B$, is a basis for $B$, we can write

$$
\begin{aligned}
b & =\sum_{b^{\prime} \in B} \lambda_{b^{\prime}} \pi\left(b^{\prime} x_{i}\right) \\
& =\sum_{b^{\prime} \in B^{(i+)}} \lambda_{b^{\prime}} b^{\prime} x_{i}+\sum_{b^{\prime} \in B-B^{(i+)}} \lambda_{b^{\prime}}\left(b^{\prime} x_{i}-u_{b^{\prime}, i}\right), \quad \lambda_{b^{\prime}} \in K,
\end{aligned}
$$


where only finitely many coefficients are $\neq 0$. Then

$$
b x_{i}^{-1}=\sum_{b^{\prime} \in B^{(i+)}} \lambda_{b^{\prime}} b^{\prime}+\sum_{b^{\prime} \in B-B^{(i+)}}\left(\lambda_{b^{\prime}} b^{\prime}-\lambda_{b^{\prime}} u_{b^{\prime}, i} x_{i}^{-1}\right), \quad \lambda_{b^{\prime}} \in K,
$$

and the induction continues as before.

To show that $B$ is indeed a Schreier basis for $R / I$ we only need to show that the elements of $B$ are linearly independent modulo $I$. But in the proof above it is shown that every non-trivial element of $I$, i.e. an element of the form $\sum u_{b, j} \alpha_{b, j}$, contains summands of positive distance from $B$, thus no non-trivial linear combination of elements of $B$ is in $I$.

(4.3) Theorem. Let $R=K\left\langle x_{1}, \ldots, x_{n}\right\rangle$, the free algebra, or the group ring of a free group of rank $n$. If $I$ is a finitely generated right ideal of $R$ there exists a finitely generated right ideal $J$ such that $I+J$ is of finite codimension and the sum is direct. Moreover, if $z$ is any element of $R-I$ then $J$ can be so chosen that $z$ is not in $I+J$. In particular, $I$ is closed in the fc-topology.

It is not hard to exhibit closed ideals that are not finitely generated and also ideals that are not closed. For example, every non-zero twosided ideal $I$ is essential, and if $R / I$ is not finite dimensional over $K$ then, by (3.4), $I$ is not finitely generated. It is easily seen that $I$ is closed if the fc-topology of $R / I$ is Hausdorff, and this is true for many ideals $I$, e.g. when $R / I$ is itself a free algebra.

On the other hand, let $I$ be a two-sided ideal in a ring $R$ such that $R / I$ is a simple ring and is not finite dimensional over $K$. Such ideals are easily available. For example, $R / I$ may be a Weyl algebra. We will show that the closure of $I$ is $R$. Denote $R / I$ by $T$. We claim first that the fc-topology of $T$ is trivial. Indeed, if $J$ is a right ideal of $T$ of finite codimension then the annihilator of $T / J$ is a twosided finite codimensional ideal of $T$, and no such exists. It follows that if $I^{\prime}$ is any open (i.e. finite codimensional) right ideal of $R$ then $I+I^{\prime}=R$. Since it is known that the closure of $I$ is the intersection of all $I+I^{\prime}$, with $I^{\prime}$ an open ideal, it follows that indeed the closure of $I$ is $R$.

Proof of (4.3). The proof in the case of the free algebra is much easier than in the case of the free group ring. Also, a closely related result appears in [8]. So we concentrate on the group ring case.

Let $I$ be a finitely generated right ideal. If $I$ is of finite codimension then there is nothing to prove. So, assume this is not the case. Let 
$B$ be a strong Schreier basis for $R / I$, relative to the basis $x_{1}, \ldots, x_{n}$ of the group (i.e. relative to the generating set $x_{1}, x_{1}^{-1}, \ldots, x_{n}, x_{n}^{-1}$ of $R$ ). As shown in (3.3) there exists $r \geq 1$ such that all group elements of length $\geq r$ that have a prefix of length $r$ which is in $B$, are also in $B$. Denote by $B_{r}$ the set of all elements of $B$ of length $\leq r$. Then for each $b \in B_{r}$ and $i=1, \ldots, n$, define $\pi^{\prime}\left(b x_{i}\right)$ to be in the linear span of $B_{r}$ such that

(i) for $b x_{i}$ of length $\leq r, \pi^{\prime}\left(b x_{i}\right)$ is the original coset representative function of $I$ : this is valid since the Schreier basis for $R / I$ is strong.

(ii) For $b x_{i}$ of length $r+1, \pi^{\prime}\left(b x_{i}\right)$ is defined such that for every fixed $i$ the set

$$
\left\{\pi^{\prime}\left(b x_{i}\right) \mid b \in B_{r}\right\}
$$

is linearly independent.

Note that since the elements $\pi^{\prime}\left(b x_{i}\right)$, for a fixed $i$, mentioned in (i) are linearly independent (by Theorem (4.2)), the whole set $\pi^{\prime}\left(b x_{i}\right)$, for a fixed $i$, is a linear basis for $\operatorname{sp}\left(B_{r}\right)$, since it is a finite linearly independent set whose cardinality is equal to the dimension of $\operatorname{sp}\left(B_{r}\right)$.

Let $J$ be the right ideal generated by the elements

$$
u_{b, i}:=b x_{i}-\pi^{\prime}\left(b x_{i}\right) \text {, }
$$

where $b \in B_{r}$, length $\left(b x_{i}\right)=r+1, i=1, \ldots, n$.

Note that the non-trivial elements of the form $b x_{i}-\pi^{\prime}\left(b x_{i}\right)$, where $b \in B_{r}$ is of length less than $r$, are a basis, over $R$, for $I$. It follows from the last theorem that $I+J$ is a right ideal of finite codimension and $B_{r}$ is a Schreier basis for $R /(I+J)$. Clearly, the sum $I+J$ is direct. Finally, if $z$ is an element of $R-I$ of length $s$, then taking $r$ to be greater than $s$ will ensure that $z$ will not be in $I+J$ (here we are using the fact that the Schreier basis is strong). By Proposition (4.1) $I$ is closed in the fc-topology.

5. Leavitt numbers. If $R$ is a ring one can define an equivalence relation $\sim$ on the set of natural numbers, according to which $p \sim q$ if the free right $R$-modules of rank $p$ and of rank $q$ are isomorphic. It is known ([7]) that this equivalence relation can be described by two integers $\mu(R), \nu(R)$, where $\mu(R)$ is allowed to be $\infty$, such that if $p<\mu(R)$ then the equivalence class of $p$ is $\{p\}$, and if $p \geq \mu(R)$ then $p \sim q$ if and only if $p \equiv q(\bmod \nu(R))$ and $q \geq \mu(R)$. If $\mu(R)=\infty$ then $R$ is said to have the unique rank property and in this case $\nu(R)$ is an irrelevant number. 
The numbers $\mu(R), \nu(R)$ are called the Leavitt numbers of $R$. Examples of rings for which the Leavitt numbers are any pair of positive integers have been given by Leavitt ([7]) and by others.

Since rings of quotients are generally thought of as "simplifying" the original object, it may appear surprising that there exist rings that have the unique rank property whose maximal ring of quotients, $Q_{\max }(R)$, and $Q_{\mathrm{fc}}(R)$ too do not have this property. In fact, it seems to be known among the experts that $Q_{\max }$ of a free ring does not have the UR property. We show that in the free case one can give precise results. Again, we work over a field $K$.

(5.1) THEOREM. If $R$ is the free algebra $K\left\langle x_{1}, \ldots, x_{n}\right\rangle \quad(n \geq 2)$ or the group ring of a free group $G$ on $n$ generators (also called $\left.x_{1}, \ldots, x_{n}\right)$ then

$$
\begin{aligned}
\mu\left(Q_{\max }(R)\right) & =1, & & \nu\left(Q_{\max }(R)\right)=1 ; \\
\mu\left(Q_{\mathrm{fc}}(R)\right) & =1, & & \nu\left(Q_{\mathrm{fc}}(R)\right)=n-1 .
\end{aligned}
$$

Throughout this proof we often write $R_{\mathscr{F}}$ for $Q_{\mathscr{F}}(R)$.

Proof. We claim, quite generally, that if a non-singular ring $R$ (e.g. a domain) contains a right ideal $J$ which is essential and free of infinite rank, ${ }^{1}$ then the Leavitt numbers of $Q_{\max }(R)$ are 1,1 . This means that we have to show that every two finitely generated free modules are isomorphic. To see that, let $J$ be such an ideal. Evidently, $J$ is isomorphic to $J \oplus J$, e.g. through any bijection between a base of $J$ and a base of $J \oplus J$ of the same cardinality. We denote the maximal localization of a module $M$ by $M_{\max }$. Then, obviously, $J_{\max } \approx J_{\max } \oplus J_{\max }$ and it remains to show that $J_{\max }$ is free of rank 1 over $Q_{\max }(R)=R_{\max }$. This, however, is a general fact: the exact sequence

$$
0 \rightarrow J \rightarrow R \rightarrow R / J \rightarrow 0
$$

gives, for every essential ideal $I$,

$$
0 \rightarrow \operatorname{Hom}_{R}(I, J) \rightarrow \operatorname{Hom}_{R}(I, R) \rightarrow \operatorname{Hom}_{R}(I, R / J) .
$$

Taking the direct limit, over $I$, gives

$$
0 \rightarrow J_{\max } \rightarrow R_{\max } \rightarrow 0
$$

since $R / J$ is a torsion module and so

$$
\underset{I}{\lim } \operatorname{Hom}_{R}(I, R / J)=0 \text {. }
$$

\footnotetext{
${ }^{1}$ In fact, many non-trivial free products of two integral domains have such ideals.
} 
Thus, $J_{\max }$ is isomorphic to $R_{\max }$ and also to $J_{\max } \oplus J_{\max }$, proving that the Leavitt numbers of $R_{\max }$ are $\mu=1, \nu=1$.

Note that the isomorphism $J_{\max } \approx R_{\max }$ does not imply that the canonical image of $J$ in $R_{\max }$ generates the unit ideal. In fact, if $J$ is not finitely generated this is not true, as follows from the considerations below, i.e. Lemma (5.2).

It remains to show that the free algebra and the free group ring (on $n \geq 2$ variables) have essential right ideals that are free of infinite rank. This follows most naturally from the fractal method of [9]. Another way is to note that every two-sided ideal $I$ of $R$ is essential as a right ideal, and if $R / I$ is not finite dimensional over $K$ then $I$ is not finitely generated, as shown in Theorem (3.3).

We now turn to $Q_{\mathrm{fc}}$. We observe, to begin with, that it is easy to see that $\mu\left(Q_{\mathrm{fc}}(R)\right)=1$ and $\nu\left(Q_{\mathrm{fc}}(R)\right)$ divides $n-1$. Indeed, $\mu$ of a ring is known to be the "smallest" rank of a free module that is not unique; and if two free modules are isomorphic then the difference of their ranks is divisible by $\nu$ of that ring. In our case, since $R$ has an ideal $I$ (the augmentation ideal) of codimension 1 which is free of rank $n$ then, as above, one proves that $I_{\mathrm{fc}} \approx R_{\mathrm{fc}}$, which gives that the free $R_{\mathrm{fc}}$ modules of rank 1 (i.e. $R_{\mathrm{fc}}$ ) and of rank $n$ (i.e. $I_{\mathrm{fc}}$ ) are isomorphic. This proves that $\mu\left(R_{\mathrm{fc}}\right)=1$ and $\nu\left(R_{\mathrm{fc}}\right)$ divides $n-1$.

Note, however, that in contrast to the case of an infinitely generated ideal, here the canonical image of $I$ in $R_{\mathrm{fc}}$ generates the unit ideal.

If $\alpha \in Q_{\mathrm{fc}}(R)$, where $R$ is as in the statement of the theorem, then $\alpha$ is represented by an element of $\operatorname{Hom}_{R}(I, R)$, for some finite codimensional ideal $I$. It is not hard to see that there is a unique "maximal right ideal of definition" for $\alpha$ : this follows since, as $R$ is an integral domain, the extension of $\alpha$ to a larger ideal is unique and so the "maximal" ideal of definition is the sum of all ideals of definition. We denote the maximal ideal of definition of $\alpha$ by $D_{\alpha}$, and its image $\alpha\left(D_{\alpha}\right)$ by $\operatorname{Im}(\alpha)$.

In what follows we repeatedly use the fact, which follows from the definitions, that if $\alpha \in Q_{\mathrm{fc}}(R)$ and $r_{1}, r_{2} \in R$, then $\alpha r_{1}=r_{2}$ in $Q_{\mathrm{fc}}(R)$ if and only if $r_{1} \in D_{\alpha}, r_{2} \in \operatorname{Im}(\alpha)$ and $\alpha\left(r_{1}\right)=r_{2}$.

(5.2) Lemma. Assume that $\alpha_{i} \in Q_{\mathrm{fc}}(R)$ ( $i$ in some index set). Then the $\alpha_{i}$ generate an essential right ideal of $Q_{\mathrm{fc}}(R)$ if and only if $\sum_{i} \operatorname{Im}\left(\alpha_{i}\right)$ is an essential right ideal of $R$.

Proof. Assume that $I=\sum_{i} \alpha_{i} Q_{\mathrm{fc}}(R)$ is an essential right ideal of $Q_{\mathrm{fc}}(R)$ and let $0 \neq r \in R$. Looking at $r$ as an element of 
$Q_{\mathrm{fc}}(R)$, there exists $\beta \in Q_{\mathrm{fc}}(R)$ such that $0 \neq r \beta \in I$. Thus, $r \beta=\sum_{j=1}^{l} \alpha_{i_{j}} \beta_{j}$, for some $l$, where $\beta_{j} \in Q_{\mathrm{fc}}(R)$. By the fact that for every $0 \neq \gamma \in Q_{\mathrm{fc}}(R)$ there exist $r_{1}, r_{2} \in R$ such that $\gamma r_{1}=r_{2} \neq 0$, it is easily seen that there is an element $s \in R$ such that $0 \neq r s=\sum_{j=1}^{l} \alpha_{i} s_{j}$ and $s_{j}, \alpha_{i} \dot{s}_{j} \in R$ for $j=1, \ldots, l$. As noted above, this implies that $\alpha_{i_{j}}\left(s_{j}\right) \in \operatorname{Im}\left(\alpha_{i}\right)$, and that $\sum_{i} \operatorname{Im}\left(\alpha_{i}\right)$ intersects non-trivially every right ideal of the form $r R$ and so is essential in $R$.

Now assume that $J=\sum_{i} \operatorname{Im}\left(\alpha_{i}\right)$ is an essential right ideal of $R$ and let $0 \neq \beta \in Q_{\mathrm{fc}}(R)$. So there exist $r_{1}, r_{2} \in R$ such that $0 \neq \beta r_{1} \in R$ and $0 \neq \beta r_{1} r_{2} \in J$. But again, as $\alpha_{i}(r)=s(r, s \in R)$ implies that $\alpha_{i} r=s$ in $Q_{\mathrm{fc}}(R)$, it follows that $\beta r_{1} r_{2} \in I=\sum_{i} \alpha_{i} Q_{\mathrm{fc}}(R)$, and $I$ is an essential right ideal.

(5.3) Lemma. Assume that $\alpha_{i} \in Q_{\mathrm{fc}}(R)$ ( $i$ in some index set) generate freely a right ideal of $Q_{\mathrm{fc}}(R)$. For each $i$ let $\left\{r_{i j}\right\}$ be a basis (over $R$ ) of the ideal of definition $D_{\alpha_{i}}$. Then the elements $\alpha_{i}\left(r_{i j}\right)$ generate freely a right ideal of $R$.

We observe that the converse of this lemma is also true (and easy to show), but we do not need it.

Proof. Suppose that

$$
\sum_{i=1}^{m} \sum_{j=1}^{t_{i}} \alpha_{i}\left(r_{i j}\right) u_{i j}=0, \quad u_{i j} \in R .
$$

Then, looking at the $\alpha_{i}$ as elements of $\operatorname{Hom}_{R}\left(D_{\alpha}, R\right)$ we get

$$
\sum_{i=1}^{m} \alpha_{i}\left(\sum_{j=1}^{t_{t}} r_{i j} u_{i j}\right)=0 \text {. }
$$

And as elements of $Q_{\mathrm{fc}}(R)$, as the $\alpha_{i}$ generate freely an ideal, it follows that

$$
\sum_{j=1}^{t_{i}} r_{i j} u_{i j}=0, \quad i=1, \ldots, m .
$$

But $D_{\alpha_{l}}$ is generated freely by the $r_{i j}$ and so $u_{i j}=0$ for all $i, j$, which gives the result.

Completion of proof of (5.1). It remains to prove that $\nu\left(Q_{\mathrm{fc}}(R)\right)=$ $n-1$. It is enough to show that if $Q_{\mathrm{fc}}(R)$, as a right $Q_{\mathrm{fc}}(R)$-module, is 
also free of rank $m \neq 1$, then $n-1$ divides $m-1$. So, let us suppose that the elements $\alpha_{1}, \ldots, \alpha_{m} \in Q_{\mathrm{fc}}(R)$ generated $Q_{\mathrm{fc}}(R)$ freely. Let $D_{\alpha_{i}}$ be the corresponding ideals of definition. Each $D_{\alpha_{i}}$ is open in the fc-topology, i.e. it is of finite codimension, say $e_{i}$. Therefore, by the Schreier-Lewin formula [8] it is free of rank $1+e_{i}(n-1)$. It follows from Lemmas (5.2) and (5.3) that $\sum_{i=1}^{m} \alpha_{i}\left(D_{\alpha_{i}}\right)$ is an essential right ideal of $R$, which is free of rank $q=m+\left(\sum_{i=1}^{m} e_{i}\right)(n-1)$. By Theorem (3.3) we get that this right ideal is of finite codimension, and again by the Schreier-Lewin formula $q \equiv 1(\bmod (n-1))$. Therefore $m \equiv 1(\bmod (n-1))$, which completes the proof.

\section{REFERENCES}

[1] P. Gabriel, Des catégories abéliennes, Bull. Soc. Math. France, 90 (1962), 323448.

[2] A. Grothendieck, Local Cohomology, (notes by R. Hartshorne), LN \#41, Springer, 1967.

[3] M. Hall, Jr. and T. Radò, On Schreier systems in free groups, Trans. Amer. Math. Soc., 64 (1948), 386-408.

[4] M. Hall, Jr., Coset representations in free groups, Trans. Amer. Math. Soc., 67 (1949), 421-432.

[5] _ A topology for free groups and related groups, Ann. of Math., (2), 52 (1950), 127-139.

[6] R. E. Johnson, The extended centralizer of a ring over a module, Proc. Amer. Math. Soc., 2 (1951), 891-895.

[7] W. G. Leavitt, Modules without invariant basis number, Proc. Amer. Math. Soc., 8 (1957), 322-328.

[8] J. Lewin, Free modules over free algebras and free group algebras: The Schreier technique, Trans. Amer. Math. Soc., 145 (1969), 455-465.

[9] A. Rosenmann and S. Rosset, Essential and inessential fractal ideals in the group ring of a free group, Bull. Amer. Math. Soc., 23 (1991), 437-442.

[10] B. Stenström, Rings of Quotients, Springer, 1975.

[11] Y. Utumi, On quotient rings, Osaka J. Math., 8 (1956), 1-18.

Received February 26, 1992 and in revised form, August 4, 1992.

TEL AVIV UNIVERSITY

TEL AVIV 69978, IsRAEL

E-mail address: aro@math.tau.ac.il 



\title{
PACIFIC JOURNAL OF MATHEMATICS
}

\author{
Founded by
}

\author{
E. F. BECKENBACH (1906-1982) F. Wolf (1904-1989)
}

\section{EDITORS}

Sun-Yung A. Chang

(Managing Editor)

University of California

Los Angeles, CA 90024-1555

chang@math.ucla.edu

\section{F. Michael Christ}

University of California

Los Angeles, CA 90024-1555

christ@math.ucla.edu

Herbert Clemens

University of Utah

Salt Lake City, UT 84112

clemens@math.utah.edu
THOMAS ENRIGHT

University of California, San Diego

La Jolla, CA 92093

tenright@ucsd.edu

Nicholas ERCOLANI

University of Arizona

Tucson, AZ 85721

ercolani@math.arizona.edu

R. FINN

Stanford University

Stanford, CA 94305

finn@gauss.stanford.edu

VAUGHAN F. R. JONES

University of California

Berkeley, CA 94720

vfr@math.berkeley.edu
SteVen KerCKHofF

Stanford University

Stanford, CA 94305

spk@gauss.stanford.edu

MARTIN ScharLemanN

University of California

Santa Barbara, CA 93106

mgscharl@math.ucsb.edu

Harold STARK

University of California, San Diego

La Jolla, CA 92093

V. S. VARADARAJAN

University of California

Los Angeles, CA 90024-1555

vsv@math.ucla.edu

\section{SUPPORTING INSTITUTIONS}

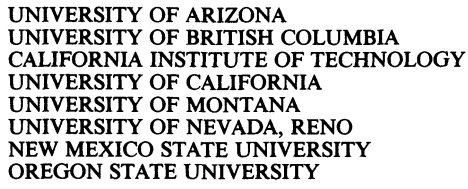

The Supporting Institutions listed above contribute to the cost of publication of this Journal, but they are not owners or publishers and have no responsibility for its content or policies.

Mathematical papers intended for publication in the Pacific Journal of Mathematics should be in typed form or offset-reproduced (not dittoed), double spaced with large margins. Please do not use built up fractions in the text of the manuscript. However, you may use them in the displayed equations. Underline Greek letters in red, German in green, and script in blue. The first paragraph must be capable of being used separately as a synopsis of the entire paper. In particular it should contain no bibliographic references. Please propose a heading for the odd numbered pages of less than 35 characters. Manuscripts, in triplicate, may be sent to any one of the editors. Please classify according to the 1991 Mathematics Subject Classification scheme which can be found in the December index volumes of Mathematical Reviews. Supply name and address of author to whom proofs should be sent. All other communications should be addressed to the managing editor, or Julie Honig, University of California, Los Angeles, California 90024-1555.

There are page-charges associated with articles appearing in the Pacific Journal of Mathematics. These charges are expected to be paid by the author's University, Government Agency or Company. If the author or authors do not have access to such Institutional support these charges are waived. Single authors will receive 75 free reprints; joint authors will receive a total of 100 free reprints. Additional copies may be obtained at cost in multiples of 50 .

The Pacific Journal of Mathematics (ISSN 0030-8730) is published monthly except for July and August. Regular subscription rate: $\$ 215.00$ a year (10 issues). Special rate: $\$ 107.50$ a year to individual members of supporting institutions.

Subscriptions, orders for numbers issued in the last three calendar years, and changes of address should be sent to Pacific Journal of Mathematics, P.O. Box 4163, Berkeley, CA 94704-0163, U.S.A. Old back numbers obtainable from Kraus Periodicals Co., Route 100, Millwood, NY 10546.

The Pacific Journal of Mathematics at University of California, c/o Department of Mathematics, 981 Evans Hall, Berkeley, CA 94720 (ISSN 0030-8730) is published monthly except for July and August. Second-class postage paid at Berkeley, CA 94704, and additional mailing offices. POSTMASTER: send address changes to Pacific Journal of Mathematics, P.O. Box 4163, Berkeley, CA 94704-0163.

PUBLISHED BY PACIFIC JOURNAL OF MATHEMATICS at University of California, Berkeley, CA 94720, A NON-PROFIT CORPORATION

This publication was typeset using $\mathcal{A} \mathcal{M} S-\mathrm{T}_{\mathrm{E}} \mathrm{X}$, the American Mathematical Society's $\mathrm{T}_{\mathrm{E}} \mathrm{X}$ macro system. Copyright (c) 1994 by Pacific Journal of Mathematics 


\section{PACIFIC JOURNAL OF MATHEMATICS}

Volume $162 \quad$ No. $2 \quad$ February 1994

On the existence of convex classical solutions to multilayer fluid

201 problems in arbitrary space dimensions

ANDREW FRENCH ACKER

Extremal functions and the Chang-Marshall inequality

VALENTIN V. ANDREEV and ALEC LANE MATHESON

Productive polynomials

RICHARD ARENS

On factor representations of discrete rational nilpotent groups and the 261 Plancherel formula

LAWRENCE JAY CORWIN and CAROLYN PFEFFER JOHNSTON

Commutants of Toeplitz operators on the Bergman space

ZELJKO CUCKOVIC

When $L^{1}$ of a vector measure is an AL-space

GUILlERMO P. CURBERA

A convexity theorem for semisimple symmetric spaces

305

KARL-HERMANN NEEB

Ideals of finite codimension in free algebras and the fc-localization

AMNON RosenmanN and ShMUEL Rosset

Dec groups for arbitrarily high exponents

BHARATH Al SETHURAMAN

Errata to: "The set of primes dividing the Lucas numbers has density $2 / 3$ "

JEFFREY C. LAGARIAS 